CNS Spectrums

www.cambridge.org/cns

\section{Letter to the Editor}

Cite this article: Naguy A, and Alamiri B (2022). Successful agomelatine monotherapy for an adolescent with attention deficit hyperactivity disorder and comorbid migraine. CNS Spectrums 27(2), 134-135.

https://doi.org/10.1017/S1092852920001923

Received: 01 October 2020

Accepted: 07 October 2020

Key words:

Agomelatine; ADHD; migraine.

*Author for correspondence:

Ahmed Naguy,

Email: ahmednagy@hotmail.co.uk (c) The Author(s), 2020. Published by Cambridge University Press.

\section{CAMBRIDGE} UNIVERSITY PRESS

\title{
Successful agomelatine monotherapy for an adolescent with attention deficit hyperactivity disorder and comorbid migraine
}

\section{Ahmed Naguy $^{1^{*}}$ (D) and Bibi Alamiri ${ }^{1,2}$}

${ }^{1}$ Almanara CAP Centre, Kuwait Centre for Mental Health (KCMH), Shuwaikh, State of Kuwait, and ${ }^{2}$ Tufts University, Medford, USA

A 15-year-old female Kuwaiti youngster was presented to our OPD clinic in accompaniment of her parents for evaluation of scholastic unattainment and behavioral disruption. She is the eldest of four siblings of nonconsanguineous monogamous family. She is the product of noncomplicated elective Caesarean section with normal developmental milestones. As a toddler, her parents report that she has always been fussy and colicky, adventurous, and curious exploring the surroundings, impersistent at toy playing and throwing excessive tantrums. During elementary school, she has always been described as overactive with frequent risk-taking behaviors, like crossing the street carelessly, paying no attention to vehicles. As getting older, she, more often than not, was noticed to be pensive and day-dreaming. Her mother always criticized her for being messy, forgetful, and a loser. She was markedly below par regarding academic tasks at school. She keeps only little company that can withstand her moodiness. Teachers always denigrate her being a bully, noisy in the classroom, and arguing too much. Remedial teaching and extra tutors could not help. There is no family of neuropsychiatric disorders. She had her menarche at age of 12 with now regular menses. No history of illicit drug use. No history of head trauma, epilepsy, or toxic exposures. No history of tics. Eye, ear, nose, and throat (EENT) evaluation was non-contributory. She has episodic migrainious headaches and is currently being maintained on prophylactic topiramate but with poor response. She was seen by a private general psychiatrist, where a diagnosis of attention deficit hyperactivity disorder (ADHD)-combined presentation was entertained and was accordingly prescribed atomoxetine $1.2 \mathrm{mg} / \mathrm{kg} / \mathrm{d}$ for 3 months with mediocre response at best, as reported by patient and parents and concordant with school records. She was then shifted to methylphenidate-ER $1 \mathrm{mg} / \mathrm{kg} / \mathrm{d}$, but the trial was prematurely aborted as she developed intolerable headaches and dysphoria nervosa. At our facility, thyroid function tests were within normal range as were iron studies. Electroencephalogram was done and unrevealing. Psychometry using Vanderbilt Assessment Scales for ADHD were contemplated, both Parents and Teacher Versions, and confirmed ADHD-combined presentation, severe type (8/9 for both subsets) with comorbid ODD (6/8 for oppositionality subset). IQ measured using Wechsler Intelligence Scale for Children-Third Edition (WISC-III) read 84 with no scatter.

After discussing with parents, a trial with agomelatine $25 \mathrm{mg} / \mathrm{d}$ was suggested. Baseline liver function tests were checked normal. Over 6 weeks, tangible improvement was appreciated both at home and school spanning behavioral, academic, and social domains. Vanderbilt was readministered and documented much lower scores (1/9 and 2/9 for inattentiveness and impulsivity/ hyperactivity subscales respectively and $2 / 6$ for oppositionality subsets). To our surprise, a parallel improvement regarding migraine control was noticed (migraine disability assessment test (MIDAS) score from baseline 22 down to 6 ) and topiramate, which is anti-cognitive, could be easily tapered off. Six months have elapsed now at writing of this report since the introduction of valdoxan and she is faring very well. Periodic follow-up of liver function tests was pursued as recommended and was perfectly normal (latest alanine transaminase (ALT) read $22 \mathrm{IU} / \mathrm{ml}$ ).

Agomelatine is a novel antidepressant with unique mechanism of action, commonly designated MASSA (melatonergic agonist specific serotonergic antagonist); M1 and M2 agonist in the suprachiasmatic nucleus with $5 \mathrm{HT} 2 \mathrm{c}$ antagonism. ${ }^{1}$ This $5 \mathrm{HT} 2 \mathrm{c}$ antagonism would translate into norepinephrine dopamine disinhibition (NDDI) in the prefrontal cortex which is mechanistically relevant to $\mathrm{ADHD}$, in theory. ${ }^{2}$ However, this is an off-label use and agomelatine is not approved as antidepressant for below age of 18 .

Similar reports have found agomelatine useful in ADHD. Recently, a double-blind randomized-controlled trial attested to the efficacy of agomelatine monotherapy for ADHD in CAP population. Fifty-four outpatients, children 6-15 years old, with DSM-IV-TR diagnosis of ADHD participated in a 6-week, parallel, double-blind, randomized clinical trial. Fifty patients completed 6 weeks of treatment with either methylphenidate (MPH) $(20 \mathrm{mg} /$ day in participants below $30 \mathrm{~kg}$ and $30 \mathrm{mg} /$ day in patients with weight $\geq 30 \mathrm{~kg}$ ) or agomelatine ( $15 \mathrm{mg} / \mathrm{d}$ in patients with weight $\geq 30 \mathrm{~kg}$ and $25 \mathrm{mg} / \mathrm{d}$ in patients with weight $\geq 45 \mathrm{~kg}$ ). Participants were assessed using Parent and Teacher ADHD Rating Scale-IV at baseline and 
at weeks 3 and 6. Changes in Teacher and Parent ADHD Rating Scale scores from baseline to the study end were not significantly different between the agomelatine group and the MPH group. Moreover, there was a trend for less insomnia in the agomelatine group versus MPH-treated group. ${ }^{3}$

Migraine has been demonstrated to be three times more frequent in children with ADHD and their mothers. Similarly, in adults, migraine was strongly associated with ADHD (odds ratio $[\mathrm{OR}]=1.8$ ) There was a significant interaction between age and gender with comorbidity increasing with age and female sex. Posthoc analysis showed that migraine with visual disturbance was generally associated with a marginally higher risk of ADHD and this was independent of ADHD endophenotypes.

Some evidence from pilot and experimental clinical trials suggests that the melatonergic system plays a role in the pathogenesis of migraine and that drugs that act on melatonin receptors may have an effect on migraine. ${ }^{4}$ Similar to our report, a case series of six patients with depression and comorbid migraine was successfully treated for both conditions with agomelatine. Agomelatine has been demonstrated to possess anti-inflammatory actions. $^{5}$

To our knowledge, this case is one of the earliest to report successful management of ADHD and comorbid migraine in CAP population using agomelatine. Agomelatine was efficacious and well tolerated. It behooves clinicians to be vigilant and mindful of ADHD-migraine comorbidity. This might open new treatment venues for such complex clinical presentations. Certainly, this finding needs replication in further large-scale studies.
Financial Support. There is no funding for this article.

Disclosures. Ahmed Naguy and Bibi Alamiri declare no conflict of interests in the past 36 months.

Authorship contributions. A.N. wrote the whole draft, assessed the case clinically and formulated the discussion. B.A. revised the whole MS, elaborated on discussion and cross-referenced it.

Ethical Approval. Ethics committee approval is not normally required in our institute for short communications.

Informed Consent: Viva voce parents' consent was obtained beforehand and patient's state of affairs was kept anonymous throughout. This has been referred to in text.

\section{References}

1. Stahl SM. Mechanism of action of agomelatine: a novel antidepressant exploiting synergy between monoaminergic and melatonergic properties. CNS Spectr. 2014;19(3):207-212.

2. Biederman J. Advances in the neurobiology of ADHD. CNS Spectr. 2007;12 (S6):6-7.

3. Naguy A, Alamiri B. Agomelatine use in child and adolescent psychiatry. Am J Ther. 2020;27(5):e556-e558. doi: 10.1097/MJT.0000000000000830.

4. Low NCP, Merikangas KR. The comorbidity of migraine. CNS Spectr. 2003; 8(6):433-434, 437-44.

5. De Berardis D, Fornaro M, Orsolini l, et al. Effect of agomelatine treatment on C-reactive protein levels in patients with major depressive disorder: an exploratory study in "real-world," everyday clinical practice. CNS Spectr. 2017;22(4):342-347. 